\title{
Spatial prediction of highway slope disasters based on Convolution Neural
}

\section{Networks}

\author{
Chao Yin ${ }^{1,2}$, Zhanghua Wang ${ }^{3}$, Xingkui Zhao ${ }^{4}$
}

(1. School of Civil and Architecture Engineering, Shandong University of Technology, Zibo, China;

2. Key Laboratory of Roads and Railway Engineering Safety Control (Shijiazhuang Tiedao University), Ministry of Education, Shijiazhuang, China;

3. Shandong Kezheng Project Management Co., LTD., Dongying, China;

4. Shandong Dongtai Engineer Consulting Co., LTD., Zibo, China)

Abstract: In order to clarify the spatial differentiations of highway slope disasters (HSDs) in Boshan District, spatial prediction was carried out based on ECG-CNN with the support of GIS. Spatial prediction factors of HSDs were selected, the stabilities of the 147 highway slopes in Boshan District were determined. The spatial prediction model of HSDs was established by ECG-CNN, and the spatial susceptibility map of HSDs in Boshan District was drawn. Influences of the prediction factor combinations and the drill sample \& verification sample combinations on the prediction success rates were verified. The results showed that low susceptible areas, medium susceptible areas and high susceptible areas account for $56.92 \%, 28.46 \%$ and $14.62 \%$ of the total areas of Boshan District respectively. Some sections of Binlai Expressway, G205, G309, S210 and S307 pass through high susceptible areas. The surface cutting depth has a small impact on the prediction success rate, while the elevation and gradient have great impacts on the prediction success rate. When the drill samples are small, network drill's maturity has a great impact on the prediction success rate, while when there are many drill samples, the model's logical structure itself has a large impact on the prediction success rate.

Keywords: Highway slope disaster (HSD); ECG-CNN; prediction factor; prediction success rate.

\section{Introduction}

Highway slope disasters (HSDs) include collapse, landslide, debris flow and slope erosion that often occur on natural or artificial slopes along highways to damage subgrade, pavement, bridges, tunnels and other structures [1]. The prevention and control of HSDs can improve the disaster resistance of highway network and accelerate the construction of "traffic power" [2]. Spatial prediction is the prerequisite for disaster monitoring and early warning based on the fusion of diverse and heterogeneous geographic, geological and hydrological information, which is of great significance to reduce economic losses and casualties [3-6]. Traditional spatial prediction models of HSDs include statistical prediction models (information quantity method, Logistic regression method, Kalman filter method, etc.) and pattern recognition models (Artificial Neural Network (ANN), Support Vector Machine (SVM), decision tree, etc.) [7-12]. Among them, ANN is widely used, however, shallow networks have problems such as local optimization, overfitting, low learning efficiency and slope diffusion, which decrease the accuracies of the prediction results to a certain degree [13,14].

Convolutional Neural Network (CNN) based on the neurocognitive machine model is a type of feed-forward neural network that includes convolutional calculations and has a deep structure [15]. As a way to overcome the shortcomings of traditional shallow networks, it has been widely used in image classification, facial recognition, audio retrieval, target location detection and other fields [16]. For instance, in order to further improve the accuracies of pavement disease statistics using two-dimensional images, Sha et al. [17] used CNN to carry out pavement disease recognition and measurement based on image classification. Zhang et al. [18] proposed a cucumber disease leaf segmentation method based on Multi-Scale Fusion Convolutional Neural Networks (MSF-CNNs), which were composed of coding networks (ENs) and decoding networks (DNs). In recent years, with the advancements of deep learning and intelligent computing researches, some scholars have introduced CNN into disaster spatial prediction. For example, Shu et al. [19] designed an automatic identification system for HSDs based on CNN, the system was developed in Caffe open source environment, integrated with the AlexNet and GoogleNet and used a large amount of highway slope data to complete the model training. Bragagnolo et al. [20] took the r.landslide database as the research object, carried out landslide susceptibility assessment based on CNN and compared the assessment results with the data published by the Brazilian Geological Survey Bureau (BGSB) to verify the accuracy of the CNN model. Wu et al. [21] used the Synthetic Minority Oversampling Technology (SMOTE) to establish the drill samples and conducted landslide susceptibility mapping (LSM) 
in Wanzhou District, Chongqing City based on CNN. Due to the short history of CNN used in disaster spatial prediction, only a few classic derivative networks (e.g., AlexNet, VGG16, GoogleNet and ResNet) have been verified for their prediction effects [22]. In contrast, researches on the use of ECG-CNN and other networks to carry out disaster spatial predictions have not been reported.

Boshan, a district in Zibo City, is located in the northern part of mountain areas of central Shandong Province. Due to the serious surface erosion and growth of gullies, the rapid development of highway construction has produced many unprotected highway slopes [23]. Combined with the severe weathering and intensification of human engineering activities, HSDs occur frequently, causing serious personal injuries and economic losses. For example, on August 3, 2015, a rockfall disaster occurred on a slope of X236, and two people were injured; on August 23, 2016, the Xiejiadian-Shiquan Highway suffered from a landslide disaster due to continuous rainfall, causing the highway to erode for more than 40 days [24]. The spatial prediction of HSDs in Boshan District, which is based on the ECG-CNN network, is to adopt the ROC (Receiver Operating Characteristic) method to explain the network structure with the highest prediction success rate, draw the susceptibility map based on GIS and verify the influences of the prediction factor combinations and the drill sample \& verification sample combinations on the prediction success rate. The aim is to provide a theoretical basis for improving highway disaster resistance and regional disaster prevention and mitigation capabilities.

\section{Investigation of HSDs in Boshan District}

\subsection{Disaster overview}

As of the end of 2020, the highway mileage of Boshan District reached 982.37km, including one expressway, two national highways and four provincial highways, the highway density was $143.26 \mathrm{~km} / 100 \mathrm{~km}^{2}$ [25]. HSDs in Boshan District are characterized by rockfall, slope erosion and slope instability. Among them, rockfalls often occur on anti-dip layered and loose broken slopes with high weathering degrees, which vary in scales and often occur suddenly. They are easy to trigger the destruction of pavement, subgrade and security facilities. Cut slopes are prone to erosion and damages due to precipitation, resulting in slope soil erosion, formation of slope gullies, slope toe erosion, road shoulder gaps, etc. The unreasonable excavation of highway slopes will destroy the natural environment's original stability and cause slope instabilities, such as avalanche, landslide and collapse, with collapse being the most common [26].

\subsection{Disaster investigation results}

HSDs are the results of the factors of disaster-pregnant environment exceeding certain thresholds. The primary task of disaster spatial prediction is to analyze the intensities, frequencies and densities of disasters in different regions, that is, to analyze the impacts of the prediction factor combinations on the probabilities and scales of disasters [27]. A comprehensive investigation of 147 highway slopes was conducted in Boshan District from October 2 to 7, 2020. The investigation results showed that the total areas of the 147 highway slopes was about $1.231 \mathrm{~km}^{2}$, the largest slope area was $0.11 \mathrm{~km}^{2}$, and the smallest slope area was $45 \mathrm{~m}^{2}$. There were 27 highway slopes with an area of more than $1,000 \mathrm{~m}^{2}$. Among the 147 highway slopes, 64 slopes are currently in a stable state, and 83 slopes are in an unstable state. The distribution of the 147 highway slopes is shown in Table 1, and some representative highway slopes are shown in Figure 1.

Table 1 Distribution of the highway slopes

\begin{tabular}{|c|c|c|c|c|c|c|c|}
\hline \multirow{2}{*}{ Highway } & \multicolumn{3}{|c|}{ Number of slopes } & \multirow{2}{*}{ Highway } & \multicolumn{3}{|c|}{ Number of slopes } \\
\hline & Stable slope & Unstable slope & Total & & Stable slope & Unstable slope & Total \\
\hline $\begin{array}{l}\text { Binlai } \\
\text { Expressway }\end{array}$ & 5 & 3 & 8 & $\begin{array}{l}\text { Xiejiadian-Shiquan } \\
\text { Highway }\end{array}$ & 3 & 8 & 11 \\
\hline G205 & 1 & 4 & 5 & S307 & 3 & 5 & 8 \\
\hline G309 & 4 & 7 & 11 & $\mathrm{X} 108$ & 10 & 16 & 26 \\
\hline S101 & 7 & 6 & 13 & $\mathrm{X} 236$ & 9 & 10 & 19 \\
\hline S105 & 3 & 5 & 8 & X302 & 9 & 5 & 14 \\
\hline S210 & 6 & 6 & 12 & X306 & 4 & 8 & 12 \\
\hline
\end{tabular}




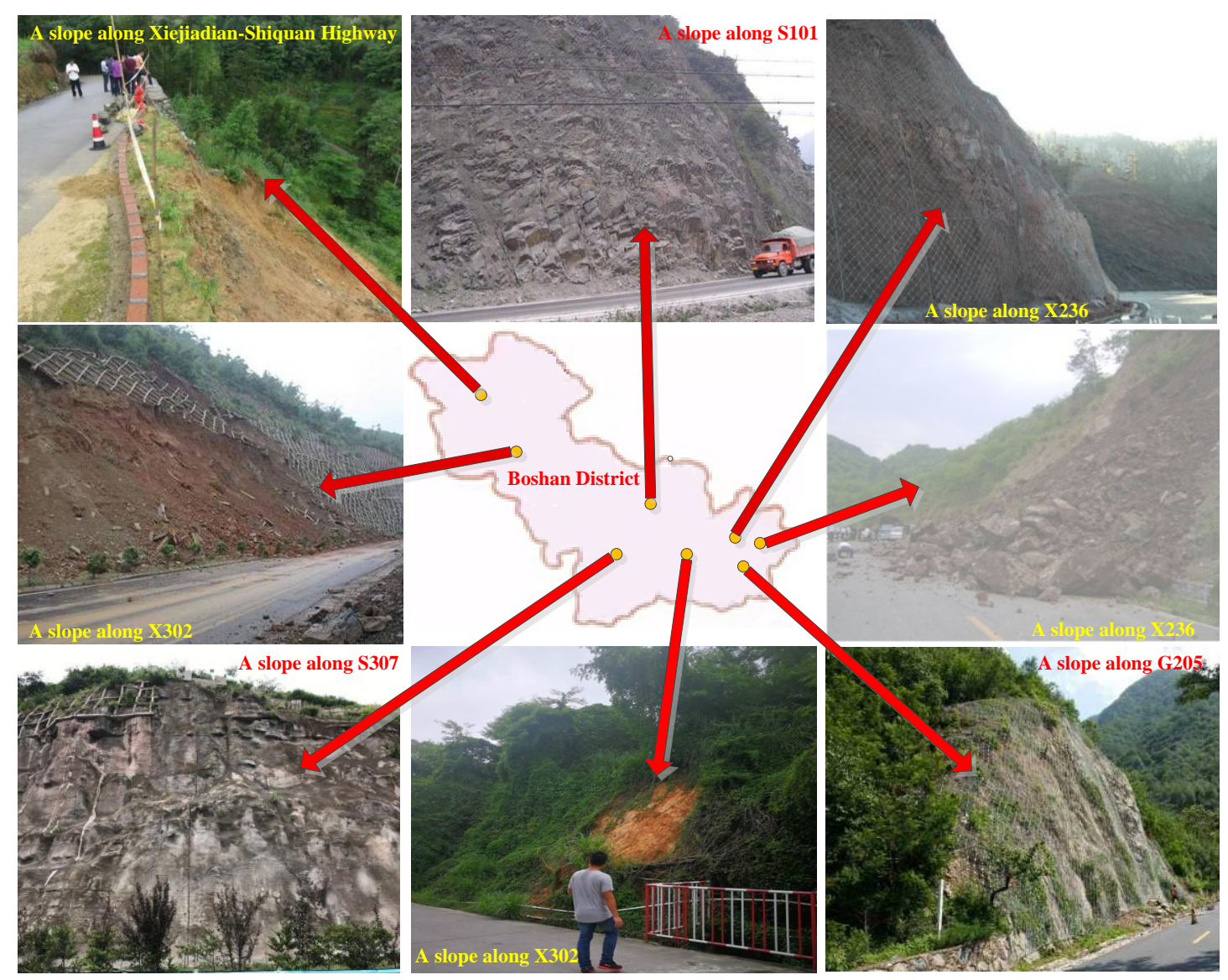

Figure 1 Representative highway slopes

\subsection{Disaster prediction factors}

According to the interaction mechanism of HSDs with the disaster-pregnant environment in the process of incubation, evolution and outbreak, the prediction factors of HSDs include topographic and geomorphic factors (elevation, gradient, surface cutting depth, surface cutting density), vegetation factor (NDVI, Normalized Difference Vegetation Index), hydrological factor (distance from river) and geotechnical factor (lithology) [28]. Although precipitation factors such as the average annual precipitation and average annual rainstorm days have significant impacts on the occurrences of HSDs [29], such factors are evenly distributed in Boshan District and have little effect on revealing the spatial differentiations of HSDs. Therefore, precipitation factors are not considered in the spatial prediction of HSDs in Boshan District.

\section{1) Topographic and geomorphic factors}

Elevation and gradient are the basic topographic and geomorphic factors, which play essential roles in HSDs. The elevations of the 147 highway slopes in Boshan District are between $162.5 \mathrm{~m}$ and $497.3 \mathrm{~m}$, and the gradients are between $25^{\circ}$ and $90^{\circ}$. Surface cutting depth and surface cutting density are important indicators to characterize the degree of surface fragmentation. Surface cutting depth is usually measured by the relative height difference of a particular catchment area, while surface cutting density is generally measured by the total length of the gullies above a certain level in a particular catchment area [30]. The method proposed by Jia et al. [31] was adopted to calculate the surface cutting depths and surface cutting densities of the 147 highway slopes. The results show that the surface cutting depths are between $9.1 \mathrm{~m}$ and $75.2 \mathrm{~m}$, and the surface cutting densities are between $0 \mathrm{~km} / \mathrm{km}^{2}$ and $0.327 \mathrm{~km} / \mathrm{km}^{2}$.

\section{2) Vegetation factor}

Vegetation roots can fix loose rocks and soils. Areas with high vegetation coverage are able to withstand weathering and reduce risks of HSDs [32,33]. NDVI is a normalized vegetation index, which can be used to reflect vegetation growth condition and spatial distribution density. The minimum value of NDVI for the 147 highway slopes is -0.649 , and the maximum value is 0.854 .

\section{3) Hydrological factor}

The closer the highway slope to the river, the higher the water content of rock and soil, the stronger the erosion, and the higher the probabilities of HSDs [34]. The minimum distance of the 147 highway slopes from river is $5 \mathrm{~m}$, and the 
maximum is $1,090 \mathrm{~m}$.

\section{4) Geotechnical factor}

Lithology provides material basis for HSDs and is one of the controlling factors of HSDs [35]. Lithology in Boshan District includes hard rock, sub-hard rock, soft rock, gravel soil, cohesive soil, sandy soil, silty soil and loess soil.

Due to the different dimensions of the prediction factors and the large differences in the data ranges, data normalization proposed by Yin et al. [2,6] was conducted before disaster spatial prediction. The details will not be repeated here.

\section{Spatial prediction models of HSDs in Boshan District}

\subsection{Building of the prediction models}

1) Structural design of the prediction models

ECG (Electrocardiogram) is a widely used clinical diagnostic feature of cardiovascular system diseases [36]. To realize the computer-aided diagnosis for cardiovascular system diseases, Zhu proposed the ECG-CNN network, which consisted of an input layer, a convolutional layer, a pooling layer, a fully connected layer and an output layer. The convolutional layer and pooling layer generally appear in pairs and can be taken several pairs [37]. The ECG-CNN network was introduced into the spatial prediction of HSDs in Boshan District. The factors that have significant impacts on the prediction success rates are the network structure depth (the number of convolutional layers and pooling layers), the number of convolutional kernels and the number of fully connected layers [38,39]. To verify the prediction success rates of different network structures, four levels of the above three factors were selected for orthogonal experimental design, and 16 ECG-CNN network structures were generated. Each network structure was calculated based on the 147 highway slope data, and the four levels selected for each factor are as follows:

(1) The network structure depth: [first level, second level, third level, fourth level] $=[1,2,3,4]$;

(2) The number of convolutional kernels: [first level, second level, third level, fourth level] $=[2,4,6,8]$;

(3) The number of fully connected layers: [first level, second level, third level, fourth level] $=[1,2,3,4]$.

The three-factor, four-level orthogonal experimental design scheme of the ECG-CNN network structures is shown in Table 2.

Table 2 Orthogonal experimental design scheme

\begin{tabular}{|l|l|l|l|}
\hline Serial number & Network structure depth & $\begin{array}{l}\text { Number of convolutional } \\
\text { kernels }\end{array}$ & $\begin{array}{l}\text { Number of fully } \\
\text { connected layers }\end{array}$ \\
\hline 1 & 1 & 8 & 4 \\
\hline 2 & 1 & 6 & 3 \\
\hline 3 & 1 & 4 & 2 \\
\hline 4 & 1 & 2 & 1 \\
\hline 5 & 2 & 8 & 3 \\
\hline 6 & 2 & 6 & 4 \\
\hline 7 & 2 & 4 & 1 \\
\hline 8 & 2 & 2 & 2 \\
\hline 9 & 3 & 8 & 2 \\
\hline 10 & 3 & 6 & 1 \\
\hline 11 & 3 & 4 & 4 \\
\hline 12 & 3 & 2 & 3 \\
\hline 13 & 4 & 8 & 2 \\
\hline 14 & 4 & 6 & 4 \\
\hline 15 & 4 & 2 & 2 \\
\hline 16 & 4 & & 3 \\
\hline
\end{tabular}

The inputs of the ECG-CNN models adopt multi-lead electrocardiographic signals, which are quite different from 
input, with the serial number of each prediction factor as the horizontal ordinate and the normalized value of each prediction factor as the vertical ordinate. For example, Figure 2 is the line chart of the prediction factors of the highway slopes \#1, \#2 and \#3. In addition, the 147 highway slopes were coded as 0 or 1 , where stable slopes were coded as 0 and unstable slopes were coded as 1 .

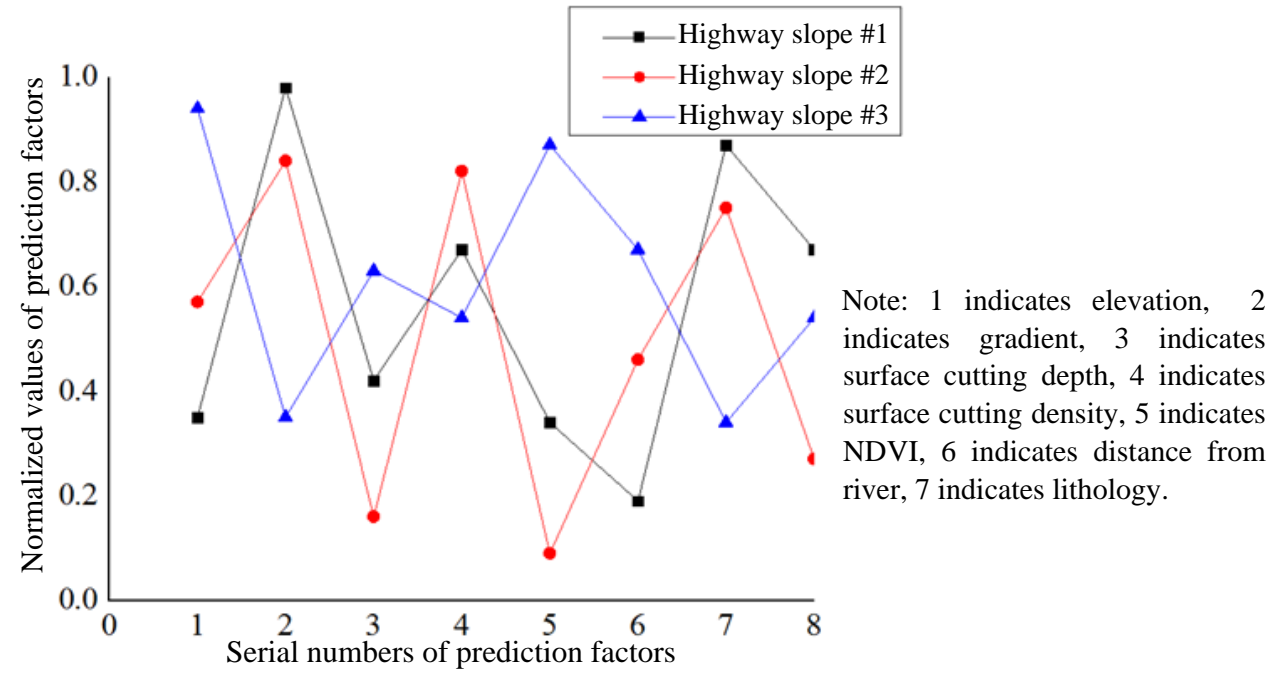

Figure 2 Line chart of the prediction factors of the highway slopes \#1, \#2 and \#3

\subsection{Verification of the prediction models}

\section{1) Computation process}

With 64 stable slopes and 21 unstable slopes being used as drill samples and the remaining 62 unstable slopes being used as verification samples, 16 ECG-CNN network structures were analyzed and computed under the following computing environment: CPU i7-6700, 8G memory, GTX1050 Ti-4G, Caffe open-source framework [40]. The following took the first structure as an example to illustrate the calculation process. The network structure included one convolutional layer $\mathrm{C} 1$, one pooling layer $\mathrm{C} 2$, and four fully connected layers F1, F2, F3 and F4. Among them, the convolutional layer $\mathrm{C} 1$ contained eight convolutional kernels, as shown in Figure 3.
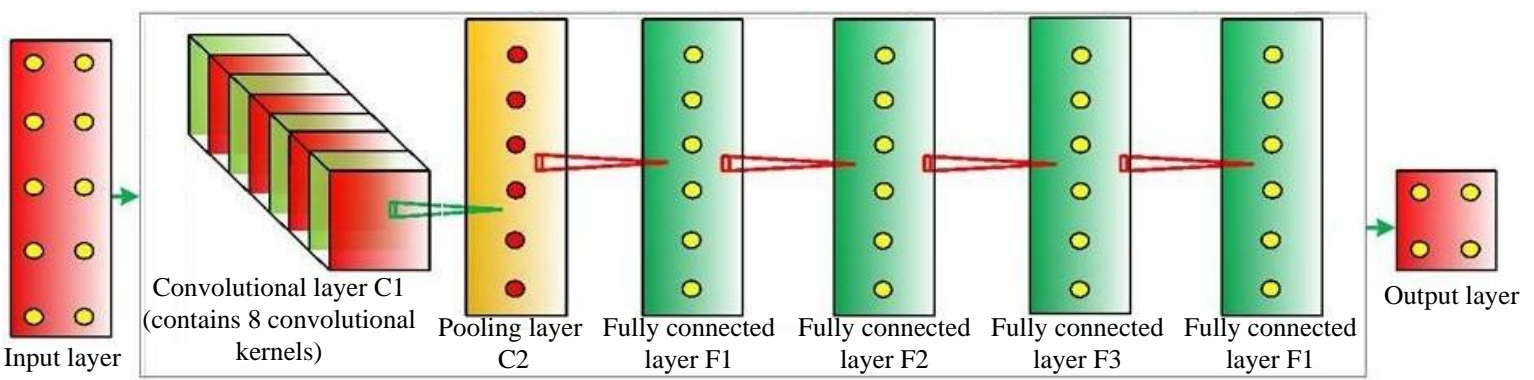

Figure 3 The first ECG-CNN network structure

Input the line charts of the 85 drill samples into the network, performed convolutional processing in the convolutional layer, and obtained the output feature matrixes through the activation function Sigmoid. The eight convolution kernels had different weights and thresholds, and the obtained output feature matrixes were also different [41]. The operation of a single convolution kernel is shown in Equation 1.

$$
x_{j}^{l}=f\left(\sum x_{j}^{l-1} W_{i}^{l}+b_{i}^{l}\right)
$$

Where: $l$ is the number of network structure layers, $i$ is the serial number of convolutional kernels, $j$ is the serial number of highway slopes, $x_{j}^{l}$ is the line chart of the $j$-th highway slope, $W_{i}^{l}$ and $b_{i}^{l}$ are the weight matrix and threshold matrix of the $i$-th convolutional kernel in the $l$-th layer, $f(x)$ is the activation function, as shown in Equation 2.

$$
f(x)=\frac{1}{1+e^{-x}}
$$

$\mathrm{C} 1$ has eight convolutional kernels, which can obtain eight disaster features. The function of the pooling layer $\mathrm{C} 2$ is to aggregate and count the above features to avoid over-fitting. The pooling operation is shown in Equation 3.

$$
x_{j}^{l+1}=\beta_{j}^{l} D\left(x_{j}^{l}\right)
$$

Where: $\beta_{j}^{l}$ is the multiplicative threshold matrix of the pooling operation, $D($ ) is the down-sampling function, which reduces the pooling feature matrix by $50 \%$ in both dimensions. The function of the fully connected layer is to establish 
the mapping relationship between the pooling aggregation features and highway slopes' stabilities so that the outputs of the stable slopes are 0 and the outputs of the unstable slopes are 1 [17]. After completing the above drill, input the 62 line charts of the verification samples into the trained network, and output the susceptible probabilities after iterative calculation. The values were in the range of 0 to 1 , where 0 meant that the disaster would not occur, and 1 meant that the disaster would occur inevitably. Set the mean square error of the prediction results of the 62 highway slopes as $M_{0}$. When $M_{0} \leqslant 0.1$, the model has better prediction ability. The computation method of $M_{0}$ is shown in Equation 4.

$$
M_{0}=\frac{\sum_{j=1}^{62}\left(O_{j}-T_{j}\right)^{2}}{62}
$$

Where: $O_{j}$ is the susceptible probability of the $j$-th highway slope, and $T_{j}$ is the stability code of the $j$-th highway slope. Since the verification samples are all unstable slopes, $T_{j}=1$. Figure 4 shows the $M_{0}$ corresponding to each iteration in the calculation process. When the number of iterations was less than $40, M_{0}$ fluctuated significantly. When the iteration reached 84 times, $M_{0}$ dropped below 0.1 , and the computation ended.

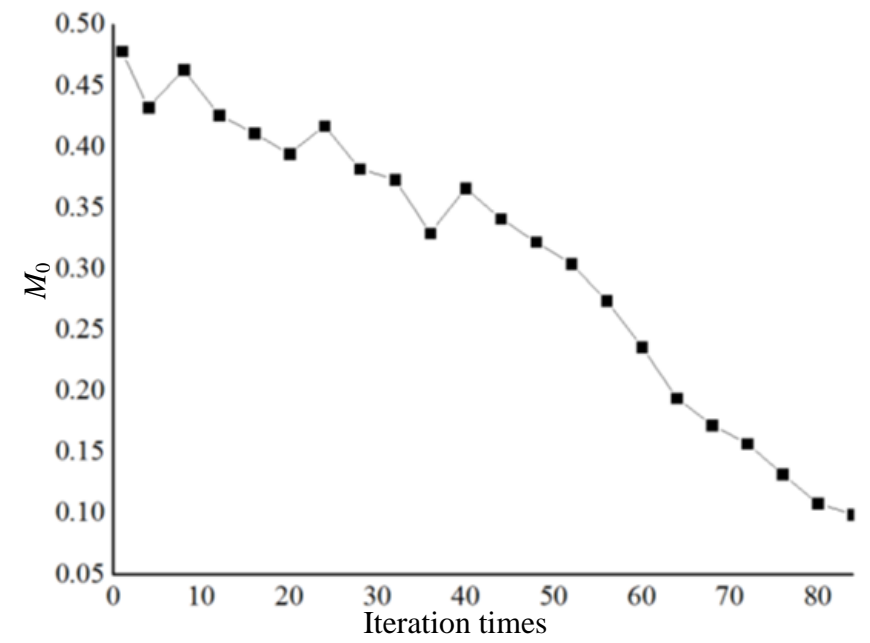

Figure 4 Relationship between the iteration times and prediction errors

2) Selection of the network

Apply the ROC method to verify each network's prediction success rate, and the prediction success rate curve of each ECG-CNN network is shown in Figure 5.
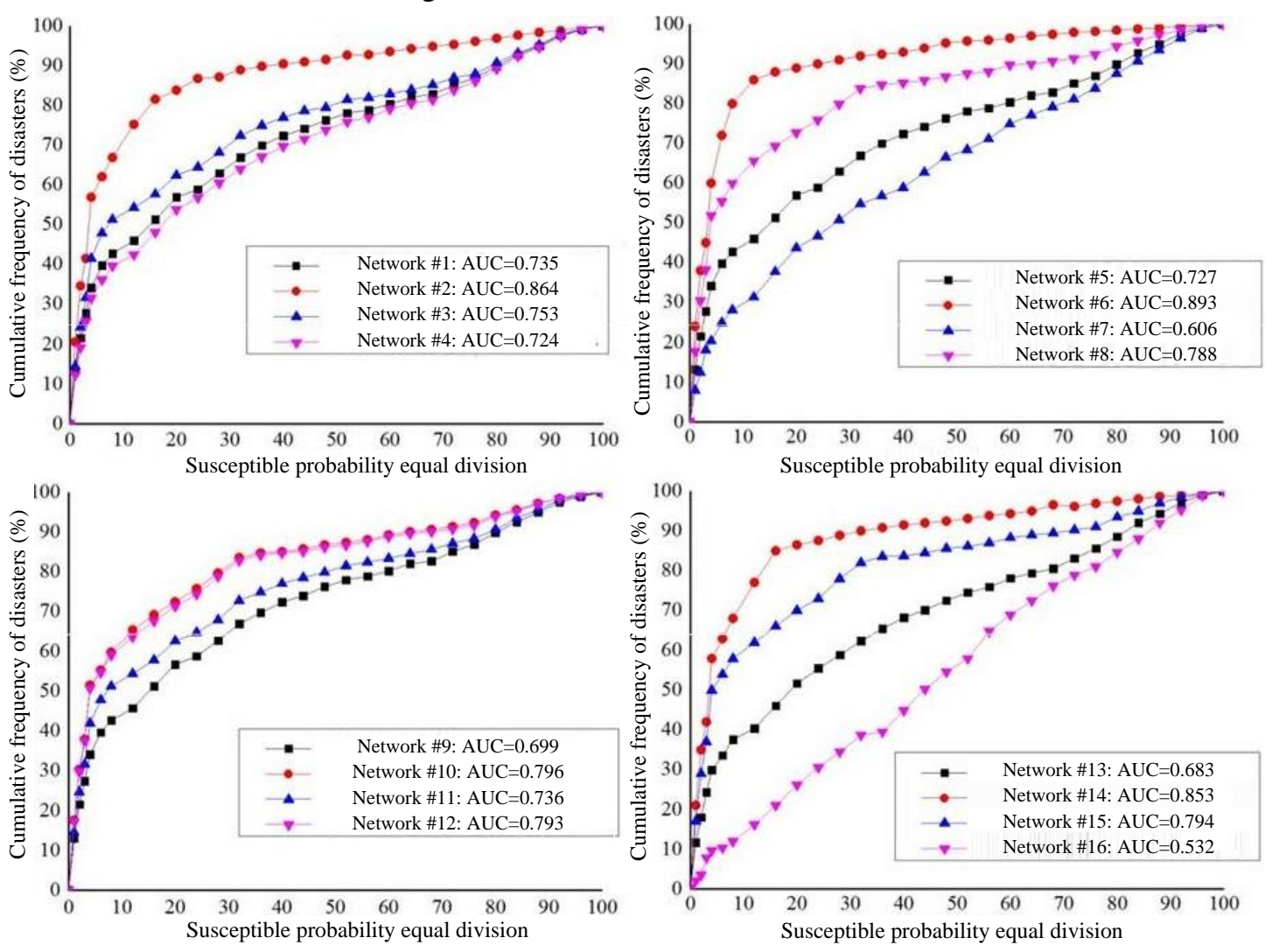
Based on Figure 5, compute the prediction success rates corresponding to different network structure depths, number of convolutional kernels and number of fully connected layers, as shown in Table 3.

Table 3 Orthogonal experimental analysis results

\begin{tabular}{|l|l|l|l|}
\hline & Network structure depths & Number of convolutional kernels & Number of fully connected layers \\
\hline First level & 0.76900 & 0.70925 & 0.70225 \\
\hline Second level & 0.75350 & 0.72225 & 0.77325 \\
\hline Third level & 0.75600 & 0.85150 & 0.79450 \\
\hline Fourth level & 0.71550 & 0.71100 & 0.72400 \\
\hline
\end{tabular}

It can be seen from Table 3 that the number of convolutional kernels has the most significant impact on the prediction success rate, followed by the number of fully connected layers, and the network structure depth has the least impact. The ECG-CNN network with the highest prediction success rate is composed of one network structure depth, six convolution kernels and three fully connected layers. The network was used to establish a spatial prediction model for HSDs in Boshan District, and 83 unstable slopes were predicted, with the success rate curve being drawn. The area under the curve was calculated to be 0.912 , as shown in Figure 6.

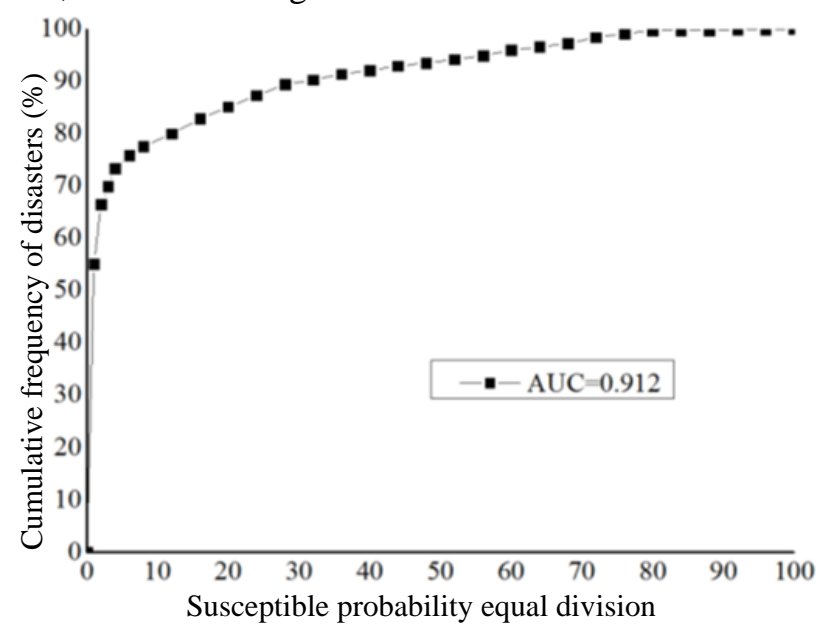

Figure 6 Prediction success rate curve

According to Shu et al. [19], when AlexNet was used for HSDs' recognition, the success rates for the four types of test sets were $73.88 \%, 86.50 \%, 87.13 \%$ and $86.38 \%$, with an average of $83.47 \%$; while the success rates of GoogleNet were $79.13 \%, 89.88 \%, 90.25 \%$ and $91.63 \%$, with an average of $87.72 \%$. According to Wu et al. [42], the accuracy rate of CNN used in evaluating landslide disaster susceptibility in Wanzhou District of the Three Gorges Reservoir was $89.50 \%$. The accuracies of these three types of networks are lower than the ECG-CNN network proposed in this study, which proves ECG-CNN's feasibility for the spatial prediction of HSDs.

\section{Spatial prediction results of HSDs in Boshan District}

Spatial prediction of HSDs in Boshan District was based on GIS, with the susceptible map being drawn. The available data included:

(1) Digital Elevation Model (DEM) in Boshan District;

(2) Digital vegetation coverage data in Boshan District;

(3) Digital precipitation data in Boshan District and its surrounding areas;

(4) Digital lithology data in Shandong Province.

For the spatial prediction of HSDs in Boshan District based on GIS, the normalized value distribution map of each prediction factor was drawn by setting the grid unit to $10 \mathrm{~m} \times 10 \mathrm{~m}$. Load the C\# language on ArcGIS Engine, and perform secondary programming development on the established prediction model. After running for about 144.5 hours, the computation of more than $6.982 \times 10^{6}$ grids were completed, and the susceptible probabilities of all grids were obtained. The results show that the largest susceptible probability is 0.963 , while the smallest is 0.082 .

According to the regional differences of the natural landforms of Boshan District and the controlling effect of each prediction factor on the occurrence of HSDs and other disaster regionalization boundaries [43,44], the susceptible 
probabilities of HSDs in Boshan District were divided into low susceptible, medium susceptible and high susceptible, and the classification boundaries were: 1) Low susceptible: [0.082, 0.357); 2) Medium susceptible: [0.358, 0.691); 3) High susceptible: $[0.692,0.963]$. According to the above classification method, the susceptible map of HSDs in Boshan

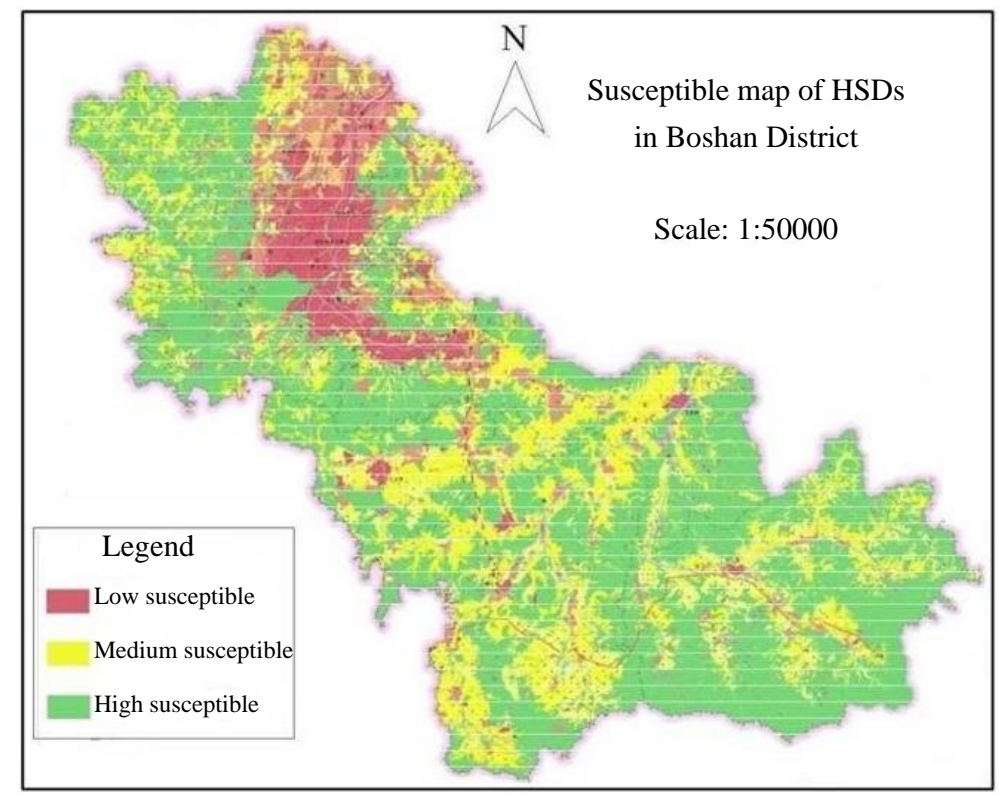

Figure 7 Susceptible map of HSDs in Boshan District

The following conclusions can be drawn from Figure 7:

1) Low susceptible, medium susceptible and high susceptible areas account for $56.92 \%, 28.46 \%$ and $14.62 \%$ of the total areas of Boshan District respectively. Among the 83 unstable slopes, 3 are located in low susceptible area, 29 are located in medium susceptible area and 51 are located in high susceptible area, accounting for $3.61 \%, 34.94 \%$ and $61.45 \%$ of the total respectively. This indicates that the spatial prediction results of HSDs in Boshan District are reasonable and correct.

2) The susceptible probabilities of HSDs in Boshan District are gradually decreasing from north to south. Apart from the apparent regional differentiations of the combination features of prediction factors, another important reason is that the engineering construction as well as population and economic density in Boshan District, that is, the damage degree to the natural environment caused by the human engineering activities decreases from north to south [45].

3) Binlai Expressway, G205, G309, S210 and S307 contain sections that pass through high susceptible areas. Therefore, in addition to strength daily maintenance, compile emergency plans and reserve emergency supplies, disaster prevention and control measures should be taken on these sections, the main measures include: (1) Carry out a comprehensive investigation of HSDs and establish a spatial database and attribute database; (2) Establish a disaster monitoring, assessment, prediction and early warning system based on the Internet of Things and rainfall forecast information, with real-time dynamic display of disaster information on GIS; 3Carry out risk assessment of HSDs, and carry out engineering protection for HSDs with unacceptable risks based on the assessment results.

\section{Discussions}

\subsection{Influences of the prediction factor combinations on the prediction success rates}

Reasonable selection of prediction factors is a prerequisite for improving prediction success rates of HSDs. Too few prediction factors will lead to a lack of disaster information and inaccurate prediction results. When there are too many prediction factors that are not completely independent, multicollinearity may occur, this makes it easy to fall into "curse of dimensionality" [46]. Five prediction factor combinations (including the combination in Section 2.3) were proposed to verify the influences of different combinations on the prediction success rates, as shown in Table 4.

Table 4 Prediction factor combinations

\begin{tabular}{|l|l|l|l|}
\hline Serial number & $\begin{array}{l}\text { Number of prediction } \\
\text { factors }\end{array}$ & Prediction factor combination & $\begin{array}{l}\text { Prediction success } \\
\text { rate }\end{array}$ \\
\hline
\end{tabular}




\begin{tabular}{|l|l|l|l|}
\hline$\# 1$ & 7 & $\begin{array}{l}\text { Elevation, Gradient, Surface cutting depth, Surface cutting } \\
\text { density, NDVI, Distance from river, Lithology }\end{array}$ & 0.912 \\
\hline$\# 2$ & 6 & $\begin{array}{l}\text { Elevation, Gradient, Surface cutting density, NDVI, } \\
\text { Distance from river, Lithology }\end{array}$ & 0.907 \\
\hline$\# 3$ & 6 & $\begin{array}{l}\text { Elevation, Surface cutting depth, Surface cutting density, } \\
\text { NDVI, Distance from river, Lithology }\end{array}$ & 0.601 \\
\hline$\# 4$ & 6 & $\begin{array}{l}\text { Elevation, Surface cutting depth, Surface cutting density, } \\
\text { NDVI, Distance from river, Lithology }\end{array}$ & 0.596 \\
\hline
\end{tabular}

Taking the 64 stable highway slopes and 21 unstable highway slopes as drill samples, line charts of the prediction factor combinations \#2 to \#5 were drew and input to the selected ECG-CNN network, and the corresponding verification samples were predicted to obtain the susceptible probability of each highway slope. ROC method was used to verify the prediction results and the prediction success rate of each combination was obtained, as shown in Figure 8.

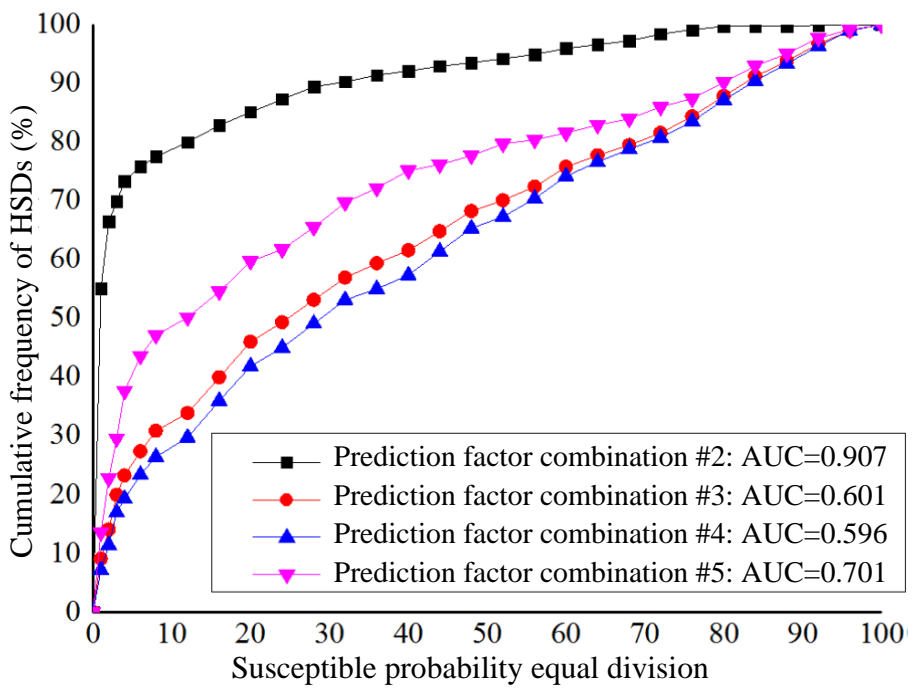

Figure 8 Prediction success rate curve of each prediction factor combination

The following conclusions can be drawn from Table 4 and Figure 8:

1) The more the prediction factors, not necessarily the better. For example, the prediction success rates of the prediction factor combinations \#3 and \#4 (6 factors) are lower than that of the prediction factor combination \#5 (5 factors);

2) The prediction success rate of prediction factor combination \#2 is only slightly lower than that of the prediction factor combination \#1, indicating that the surface cutting depth has little effect on the prediction success rate;

3) The prediction success rates of the prediction factor combinations \#3 and \#4 are low, indicating that the elevation and gradient factors have more significant impacts on the prediction success rate.

\subsection{Influences of the drill sample $\&$ verification sample combinations on the prediction success rates}

Drill sample \& verification sample combinations have great impacts on the studding abilities of ECG-CNN networks. Four sets of drill sample \& verification sample combinations (including the combination in Section 3.2) were proposed to verify their prediction success rates respectively, as shown in Table 5.

Table 5 Drill sample \& verification sample combinations

\begin{tabular}{|l|l|l|l|}
\hline Serial number & Drill samples & Verification samples & Prediction success rate \\
\hline$\# 1$ & 64 stable slopes, 21 unstable slopes & 62 unstable slopes & 0.912 \\
\hline$\# 2$ & 64 stable slopes, 35 unstable slopes & 48 unstable slopes & 0.915 \\
\hline$\# 3$ & 50 stable slopes, 15 unstable slopes & 68 unstable slopes & 0.804 \\
\hline$\# 4$ & 40 stable slopes, 8 unstable slopes & 75 unstable slopes & 0.535 \\
\hline
\end{tabular}

Line charts of the drill sample \& verification sample combinations \#2 to \#4 were proposed and input to the selected ECG-CNN network, the corresponding verification samples were predicted to obtain the susceptible probability of each 
highway slope. ROC method was used to verify the prediction results and the prediction success rate of each combination was obtained, as shown in Figure 9.

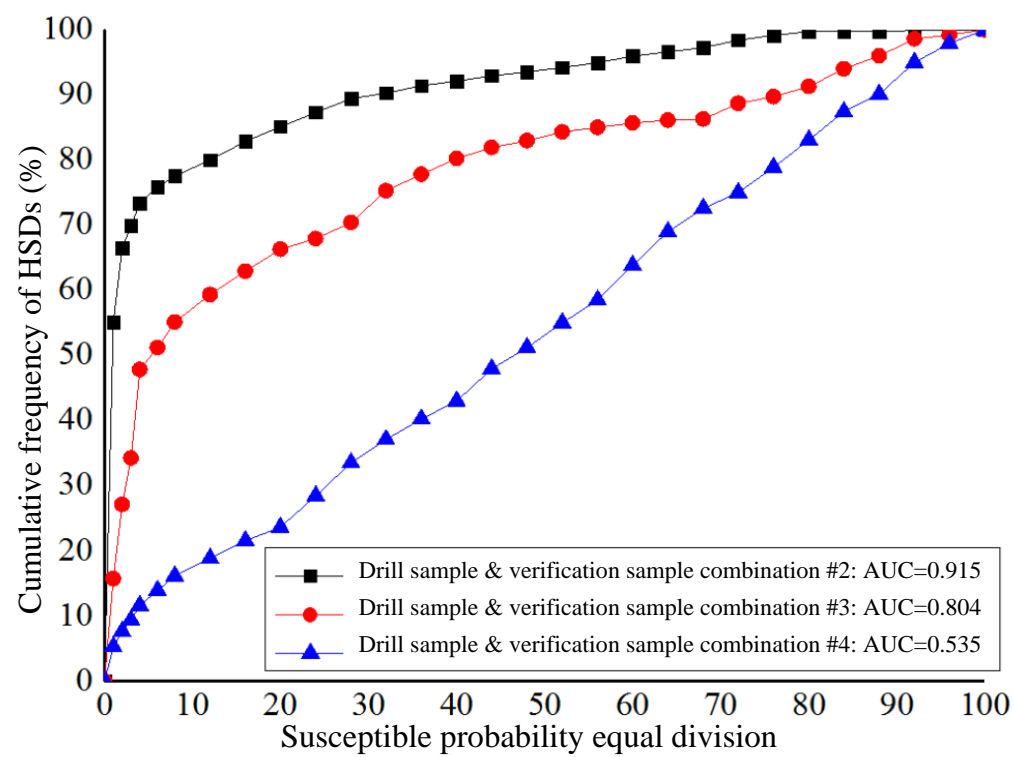

Figure 9 Prediction success rate curve of each drill sample \& verification sample combination

The following conclusions can be drawn from Table 5 and Figure 9:

1) When the drill samples increase, the prediction success rate increases, but the magnitude is not large, indicating that the network drill has matured at this time, and the prediction error is caused by the limitation of the model's logical structure itself;

2) When the drill samples decrease, the prediction success rate decreases significantly, indicating that network drill's maturity has a larger impact on the prediction success rate.

\section{Conclusions}

1) Spatial prediction factors of HSDs were selected and spatial prediction models were built on the basis of ECG-CNN in Boshan District. Susceptible map of HSDs in Boshan District was plotted on the basis of GIS. The results show that low susceptible areas, medium susceptible areas and high susceptible areas account for $56.92 \%, 28.46 \%$ and $14.62 \%$ of the total areas of Boshan District respectively. Some sections of Binlai Expressway, G205, G309, S210 and S307 pass through high susceptible areas.

2) Influences of the prediction factor combinations and the drill sample \& verification sample combinations on the prediction success rates were verified. The results show that the surface cutting depth has a small impact on the prediction success rate, while the elevation and gradient have great impacts on the prediction success rate. When the drill samples are small, network drill's maturity has a great impact on the prediction success rate, while when there are many drill samples, the model's logical structure itself has a large impact on the prediction success rate.

\section{Acknowledgement:}

This research is supported by the National Natural Science Foundation of China (Grant NO. 51808327) and Natural Science Foundation of Shandong Province (Grant NO. ZR2019PEE016).

\section{Ethical statements:}

I certify that this manuscript is original and has not been published and will not be submitted elsewhere for publication while being considered by Natural Hazards. And the study is not split up into several parts to increase the quantity of submissions and submitted to various journals. No data have been fabricated or manipulated (including images) to support our conclusions. No data, text or theories by others are presented as if they were our own. The submission has been received explicitly from all co-authors. Authors whose names appear on the submission have contributed sufficiently to the scientific work and therefore share collective responsibility and accountability for the results.

\section{References:}


[1] Li YJ, Xie QL (2013) Study on discriminant criterion of highway landslide disaster. Applied Mechanics \& Materials 275-277:27352739.

[2] Yin C, Li HR, Che F, Li Y, Hu ZN, Liu D (2020) Susceptibility mapping and zoning of highway landslide disasters in China. PLOS ONE 15(9):0235780.

[3] Sezer EA, Nefeslioglu HA, Osna T (2017) An expert-based landslide susceptibility mapping (LSM) module developed for Netcad Architect Software. Computers \& Geosciences 98:26-37.

[4] Hong HY, Liu JZ, Bui DT, Pradhan B, Acharya TD, Pham BT, Zhu AX, Chen W, Ahmad BB (2018) Landslide susceptibility mapping using J48 Decision Tree with AdaBoost, Bagging and Rotation Forest ensembles in the Guangchang area (China). CATENA 163:399413.

[5] Wang Y, Duan HX, Hong HY (2019) A comparative study of composite kernels for landslide susceptibility mapping: A case study in Yongxin County, China. CATENA 183:104217.

[6] Yin C (2020) Hazard assessment and regionalization of highway flood disasters in China. Natural Hazards 100:535-550.

[7] San BT (2014) An evaluation of SVM using polygon-based random sampling in landslide susceptibility mapping: The Candir catchment area (western Antalya, Turkey). International Journal of Applied Earth Observation and Geoinformation 26:399-412.

[8] Zhou C, Yin KL, Cao Y, Ahmed B (2016) Application of time series analysis and PSO-SVM model in predicting the Bazimen landslide in the Three Gorges Reservoir, China. Engineering Geology 204:108-120.

[9] Chen W, Pourghasemi HR, Kornejady A, Zhang N (2017) Landslide spatial modeling: Introducing new ensembles of ANN, MaxEnt, and SVM machine learning techniques. Geoderma 305:314-327.

[10] Zhu AX, Miao YM, Yang L, Bai SB, Liu JZ, Hong HY (2018) Comparison of the presence-only method and presence-absence method in landslide susceptibility mapping. CATENA 171:222-233.

[11] Ali SA, Parvin F, Vojteková J, Comulus R, Linh NTT, Pham QB, Vojtek M, Gigovic L, Ahmad A, Ghorbani MA (2021) GIS-based landslide susceptibility modeling: A comparison between fuzzy multi-criteria and machine learning algorithms. Geoscience Frontiers 12(2):857-876.

[12] Sun DL, Xu JF, Wen HJ, Wang DZ (2021) Assessment of landslide susceptibility mapping based on Bayesian hyperparameter optimization: A comparison between Logistic regression and random forest. Engineering Geology 281:105972.

[13] Cui P, Xiang LZ, Zou Q (2013) Risk assessment of highways affected by debris flows in Wenchuan earthquake area. Journal of Mountain Science 10(2):173-189.

[14] Yang JT, Song C, Yang Y, Xu CD, Guo F, Xie L (2019) New method for landslide susceptibility mapping supported by spatial logistic regression and GeoDetector: A case study of Duwen Highway Basin, Sichuan Province, China. Geomorphology 324:62-71.

[15] Kiranyaz S, Ince T, Gabbouj M (2016) Real-time patient-specific ECG classification by 1D Convolution Neural Networks. IEEE Transactions on Biomedical Engineering 63(3):664-675.

[16] Li X, Jie ZQ, Feng JS, Liu CS, Yan SC (2018) Learning with rethinking: Recurrently improving Convolutional Neural Networks through feedback. Pattern Recognition 79:183-194.

[17] Sha AM, Tong Z, Gao J (2018) Recognition and measurement of pavement disasters based on Convolutional Networks. China Journal of Highway Transport 31(1):1-10. (in Chinese)

[18] Zhang SW, Wang Z, Wang ZL (2020) Method for image segmentation of cucumber disease leaves based on multi-scale fusion Convolutional Neural Networks. Transactions of the Chinese Society of Agricultural Engineering 36(16):149-157. (in Chinese)

[19] Shu JX, Zhang JL, Wu JT (2017) Research on identification of slope disasters along highways based on deep Convolution Neural Network. Highway Transport Application Technology 154:70-74. (in Chinese)

[20] Bragagnolo L, Silva RV, Grzybowski JMV (2020) Landslide susceptibility mapping with r.landslide: A free open-source GISintegrated tool based on Artificial Neural Networks. Environmental Modelling and Software 123:104565.

[21] Wu XL, Yang JY, Niu RQ (2020) A landslide susceptibility assessment method using SMOTE and Convolutional Neural Network. Geomatics and Information Science of Wuhan University 45(8):1223-1232. (in Chinese)

[22] Sahin EK, Colkesen I, Acmali SS, Akgun A, Aydinogu AC (2020) Developing comprehensive geocomputation tools for landslide susceptibility mapping: LSM tool pack. Computers \& Geosciences 144:104592.

[23] Peethambaran B, Anbalagan R, Kanungo DP, Goswami A, Shihabudheen KV (2020) A comparative evaluation of supervised machine learning algorithms for township level landslide susceptibility zonation in parts of Indian Himalayas. CATENA 195:104751.

[24] Sun Q, Shi QM (2020) Study on the risk zoning of urban earthquake disaster based on GIS: Take Zibo City as an example. Earthquake 
Research in Sichuan 2:19-24. (in Chinese)

[25] Jiang C, Qu BJ, Dong Q, Lin MS (2014) Construction of Geological Disasters Warning System Based on GIS in Zibo City. Shandong Land and Resource 30(3):89-91. (in Chinese)

[26] Zhu T, Zhou J, Wang H (2017) Localization and characterization of the Zhangdian-Renhe fault zone in Zibo city, Shandong province, China, using electrical resistivity tomography (ERT). Journal of Applied Geophysics 136:343-352.

[27] Yin C, Zhang JL (2018) Hazard regionalization of debris-flow disasters along highways in China. Natural Hazards 91:129-147.

[28] Sun DL, Shi SX, Wen HJ, Xu JH, Zhou XZ, Wu JP (2021) A hybrid optimization method of factor screening predicated on GeoDetector and Random Forest for Landslide Susceptibility Mapping. Geomorphology 379:107623.

[29] Yi YN, Zhang ZJ, Zhang WC, Jia HH, Zhang JQ (2020) Landslide susceptibility mapping using multiscale sampling strategy and convolutional neural network: A case study in Jiuzhaigou region. CATENA 195:104851.

[30] Li ZQ, Allegre O, Li QL, Guo W, Li L (2021) Femtosecond laser single step, full depth cutting of thick silicon sheets with low surface roughness. Optics \& Laser Technology 138:106899.

[31] Jia XL, Xu JL, Yang HZ, Zhao LP (2012) Calculation of broken index of surface based on GIS. Journal of Chongqing University 35(11):126-130. (in Chinese)

[32] Ghebrezgabher MG, Yang TB, Yang XM, Sereke TE (2020) Assessment of NDVI variations in responses to climate change in the Horn of Africa. The Egyptian Journal of Remote Sensing and Space Science 23(3):249-261.

[33] Jiang R, Sanchez-Azofeifa A, Laakso K, Wang P, Xu Y, Zhou ZY, Luo XW, Lan YB, Zhao GP, Chen X (2021) UAV-based partially sampling system for rapid NDVI mapping in the evaluation of rice nitrogen use efficiency. Journal of Cleaner Production 289:125705.

[34] Dehnavi A, Aghdam IN, Pradhan B, Varzandeh MHM (2015) A new hybrid model using step-wise weight evaluation ratio analysis (SWARA) technique and adaptive neuro-fuzzy inference system (ANFIS) for regional landslide hazard evaluation in Iran. CATENA, 135:122-148.

[35] Dou J, Yunus AP, Bui DT, Merghadi A, Sahana M, Zhu ZF, Chen CW, Khosravi K, Yang Y, Pham BT (2019) Assessment of advanced random forest and decision tree algorithms for modeling rainfall-induced landslide susceptibility in the Izu-Oshima Volcanic Island, Japan. Science of The Total Environment 662:332-346.

[36] Li RF, Hou CL, Zhou H, Dai YS, Jin LQ, Xi Q (2020) Comparison on radiation effective dose and image quality of right coronary artery on prospective ECG-gated method between 320 row CT and 2nd generation (128-slice) dual source CT. Journal of Applied Clinical Medical Physics 21(8):1-7.

[37] Zhu HH. Key algorithms on computer-aided electro-cardiogram analysis and development of remote multi-signs monitoring system. (Doctor Thesis) Suzhou Institute of Nano-tech and Nano-bionics, Chinese Academy of Sciences, Suzhou, Jiangsu, China, 2013.

[38] Zhang MK, Xu L, Xiong J, Zhang XD (2020) Correlation filter via random-projection based CNNs features combination for visual tracking. Journal of Visual Communication and Image Representation 77:103082.

[39] Zeng LC, Sun B, Zhu DQ (2021) Underwater target detection based on Faster R-CNN and adversarial occlusion network. Engineering Applications of Artificial Intelligence 100:104190.

[40] Zhang KK, Wu QF, Chen YP (2021) Detecting soybean leaf disease from synthetic image using multi-feature fusion faster R-CNN. Computers and Electronics in Agriculture 183:106064.

[41] Xie J, Hu K, Li GF, Guo Y (2021) CNN-based driving maneuver classification using multi-sliding window fusion. Expert Systems with Applications 169:114442.

[42] Kumar D, Thakur M, Dubey CS, Shukla DP (2017) Landslide susceptibility mapping \& prediction using Support Vector Machine for Mandakini River Basin, Garhwal Himalaya, India. Geomorphology 295:115-125.

[43] Huang Y, Zhao L (2018) Review on landslide susceptibility mapping using support vector machines. CATENA 165:520-529.

[44] Zhang GL, Wang M, Liu K (2019) Forest Fire Susceptibility Modeling Using a Convolutional Neural Network for Yunnan Province of China. International Journal of Disaster Risk Science 10(3):386-403.

[45] Gao XM, Qin ZL, Wang LJ, Chen LX, Ma SQ, Yang KD (2012) The climatic characteristics of geological calamity in the mountainous area of the middle part of Shandong Province. Science \& Technology Review 30(4):55-60. (in Chinese)

[46] Wen Q, Xia LG, Li LL, Wu W (2013) Automatically samples selection in disaster emergency oriented land-cover classification. Geomatics and Information Science of Wuhan University 38(7):799-804. (in Chinese) 